\title{
Rapid Access to Intervention Development
}

National Cancer Institute

\section{Source}

National Cancer Institute. Rapid Access to Intervention Development. NCI Thesaurus.

Code C19472.

RAID is a new program designed to facilitate translation to the clinic of novel, scientifically meritorious therapeutic interventions originating in the academic community. It will do this by making available to the academic research community, on a competitive basis, $\mathrm{NCl}$ resources for the pre-clinical development of drugs and biologics. 